\title{
O Terapeuta Ocupacional na Atençáo Básica em Saúde: a representatividade em revistas e nos congressos brasileiros da área ${ }^{1}$
}

\author{
Bianca Gonçalves de Carrasco Bassi ${ }^{a}$, Ana Paula Serrata Malfitano ${ }^{\text {, }}$ \\ Pamela Cristina Bianchi ${ }^{\mathrm{c}}$
}

\author{
${ }^{a}$ Mestre em Terapia Ocupacional, Programa de Pós-graduação em Terapia Ocupacional, \\ Especialista em Saúde da Família, Universidade Federal de São Carlos - UFSCar \\ bDoutora em Saúde Pública, Universidade de São Paulo - USP, Professora Adjunta do \\ Departamento de Terapia Ocupacional e do Programa de Pós-graduação em Terapia Ocupacional, \\ Universidade Federal de São Carlos - UFSCar

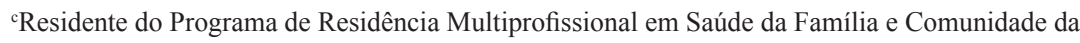 \\ Universidade Federal de São Carlos - UFSCar, Graduada em Terapia Ocupacional, \\ Bolsista do Programa PIBIC/CNPq/UFSCar 2010-2011
}

\begin{abstract}
Resumo: Nos anos 2000, impulsionadas pela Estratégia Saúde da Família e pelos investimentos governamentais em seu entorno, vimos emergirem reflexões ascendentes sobre a Atenção Primária em Saúde (APS), no Brasil chamado de Atenção Básica à Saúde (ABS). Como parte integrante de nossas pesquisas nesse âmbito, colocamos o foco sobre a questão da atuação profissional terapêutico-ocupacional nessa área. Neste artigo objetivamos apresentar um mapeamento da discussão da categoria acerca de sua intervenção nesse contexto, elegendo para tal os dois periódicos nacionais de maior circulação entre os terapeutas ocupacionais, assim como o fórum nacional de maior importância para a categoria: os congressos brasileiros de Terapia Ocupacional. Buscamos artigos publicados na temática no período de 2000 a 2011, assim como o conhecimento científico apresentado nos últimos sete congressos, no período de 1999 a 2011. Encontramos 21 artigos completos publicados sobre a temática nas revistas científicas especificadas no período. A análise dos artigos mostrou que a maioria referia-se à atuação do terapeuta ocupacional junto às pessoas com deficiência na ABS e principalmente resultados advindos de ações de pesquisa e ensino ligadas às universidades paulistas. Com relação aos trabalhos apresentados nos congressos, dos 3.755 trabalhos apresentados no período de 1999 a 2011, 191 deles referiam-se à Atenção Básica em Saúde, ou seja, cerca de 5\%. Os dados revelam uma ascendência de discussões da categoria sobre essa temática no período estudado. Conclui-se que o tema vem sendo enfocado no interior da profissão, requerendo o aprofundamento de pesquisas que se dediquem à produção de conhecimento nesse campo.
\end{abstract}

Palavras-chave: Publicações de Divulgação Científica, Terapia Ocupacional, Atenção Primária à Saúde.

\section{The occupational therapist in Primary Health Care: representation in journals and Brazilian congresses}

\begin{abstract}
In the 2000's, supported by governmental investments in the Family Health Strategy, reflections on the subject of Primary Health Care (PHC) began to arise, which in Brazil was called Basic Health Care. As part of the research in the Primary Health Care matter, an analysis on the occupational therapy work in this context was carried out. This article seeks to present a discussion map of the category about its intervention in the area based in two national Journals of Occupational Therapy and the main local forum of discussion, the Brazilian
\end{abstract}


Congress of Occupational Therapy. Articles with this thematic published between 2000 and 2011, as well as the scientific knowledge presented in the last seven congresses (1999-2011) were searched. Twenty-one full articles on this theme published in specialized Periodicals during this period were selected. The investigation showed that most articles related to the assistance of the occupational therapist to disabled people in Primary Health Care, mainly results of research and education studies carried out by universities from the State of Sao Paulo. With respect to the papers presented in the congresses, from a total of 3755, $191(5 \%)$ scientific congress presentations concerned Primary Health Care. Results showed an increase in the discussions on this theme during the study period. It was possible to conclude that more importance has been given to this theme and more comprehensive researches are needed to support knowledge improvement in this field.

Keywords: Publications for Science Dissemination, Occupational Therapy, Primary Health Care.

\section{Introdução}

Desde os anos 1930 são desenvolvidas experiências de medicina comunitária, sendo que, a partir de 1978, a Atenção Primária em Saúde (APS) é destaque mundial, permanecendo temática central de discussão e elaboração de estratégias a sua ampliação. Tem-se um marco em Alma Ata, na I Conferência Internacional de Cuidados Primários de Saúde, realizada pela Organização Mundial de Saúde (OMS), momento em que se estabeleceu como prioritária essa modalidade de assistência em saúde (CASTRO, 2009).

No Brasil, com a instituição do Sistema Único de Saúde (SUS), foi garantido a todos os brasileiros $o$ atendimento universal, integral e equânime em saúde. A chamada Atenção Básica em Saúde ${ }^{2}$ (ABS) ganhou destaque após a criação do SUS, sendo considerada a base estruturante do sistema para se atingir a utopia sanitarista, a qual previa que entre $70 \%$ e $80 \%$ dos problemas de saúde da população deveriam ser resolvidos próximos à sua residência, ou seja, no nível primário de atenção (CAMPOS, 1989).

Como estratégia prioritária para o fortalecimento das açôes da Atenção Básica em Saúde no SUS, ocorreu na década de 1990 a criação do Programa de Agentes Comunitários (PACS), seguido pela implantação do Programa de Saúde da Família (PSF), atualmente reconhecido como Estratégia Saúde da Família (ESF) em todo o país (BRASIL, 2010a).

Em 2006 foi apresentada a Política Nacional de Atenção Básica (PNAB) pelo Ministério da Saúde, estabelecendo os princípios que vêm norteando a organização dos serviços: o trabalho em equipe, a territorialização, a corresponsabilidade sanitária, o foco nas necessidades da população e a unidade básica de saúde da família como porta de entrada preferencial dos usuários no sistema de saúde.

A partir de 2008, com a criação dos Núcleos de Apoio à Saúde da Família (NASF), o Ministério da Saúde propôs ampliar a abrangência e o escopo das açôes da ABS, especialmente das ESF, bem como sua resolubilidade, apoiando a inserção na rede de serviços, além do processo de territorialização e de regionalização a partir desse nível de atenção à saúde (BRASIL, 2010b).

Esse cenário trouxe uma ampliaçáo de espaços de intervenção para o terapeuta ocupacional, fomentando o desenvolvimento da profissão naquele âmbito assistencial do cuidado em saúde. Ao final da década de 1990, terapeutas ocupacionais passaram a atuar em maior número na $\mathrm{ABS}$, com propostas de atenção integral através de açôes de prevenção, promoção e reabilitação, em consonância com as diretrizes políticas vigentes, as quais injetaram recursos financeiros para induzir a Estratégia Saúde da Família (ESF) como modelo de reestruturação da ABS no país (MOREIRA, 2008). A inserção da profissão junto a novas demandas populacionais, novos campos e núcleos de atuação se volta cada vez mais para espaços extrainstitucionais, com intervençóes no território e na comunidade (ALMEIDA; OLIVER, 2001).

Assim, como parte integrante de nossas pesquisas no contexto da $\mathrm{ABS}$, focamos a análise na questão da atuação profissional terapêutico-ocupacional nesse contexto. De acordo com dados de Caldeira (2009), a temática mostra-se relevante pela crescente incorporação desse profissional nesse nível de cuidado em saúde. Para a autora, a inserção de terapeutas ocupacionais em Unidades Básicas de Saúde (UBS) tem se ampliado significativamente. Ela é resultante de um processo indutório do Ministério da Saúde com direção de financiamento para a ABS, tendo como objetivo declarado a facilitaçáo do acesso das populaçóes ao sistema de saúde e a ampliaçáo da integralidade do cuidado.

No âmbito de tal discussão, objetivamos aqui apresentar um mapeamento dos debates da categoria acerca de sua intervenção nessa área, elegendo para tal os dois periódicos nacionais de maior circulação na área, a saber: Cadernos de Terapia Ocupacional da UFSCar e Revista de Terapia Ocupacional da USP; assim como o fórum nacional de maior importância e circulação entre profissionais e pesquisadores do país: os congressos brasileiros de Terapia Ocupacional (CBTO). 


\section{Metodologia}

Com o intuito de conhecer a discussão registrada pela categoria sobre a intervenção na $A B S$, pesquisamos artigos publicados no período de 2000 a 2011 nos Cadernos de Terapia Ocupacional da UFSCar e na Revista de Terapia Ocupacional da USP. Assinala-se que, referente ao ano de 2011, a Revista de Terapia Ocupacional da USP foi pesquisada apenas no volume 1 e 2, pois não estava disponível, até o início de 2012, o volume 3 referente àquele ano. Vale destacar que esses dois periódicos são os únicos de circulação nacional na área que mantêm periodicidade e reúnem difusão de conhecimento e de experiências em Terapia Ocupacional no país. Tivemos acesso aos artigos por meio das publicaçôes de cada periódico, disponibilizadas nos formatos impresso e eletrônico. Ver a lista de periódicos estudados no Quadro 1.

Optamos também por conhecer as produçôes da categoria apresentados nos últimos sete (7) congressos brasileiros de Terapia Ocupacional (CBTO), no período de 1999 a 2011, por meio dos anais de cada evento. Destacamos que esses congressos tiveram organizaçóes distintas e também diferentes formas de divulgação, conforme Quadro 2.

Utilizamos como critério de seleção e classificação dos artigos e dos trabalhos apresentados nos CBTOs, as seguintes proposiçóes: Pesquisas ou trabalhos sobre a atuação do terapeuta ocupacional inserido na ABS; Trabalhos em que Unidades Básicas de Saúde e/ou Unidades de Saúde da Família foram local da pesquisa e/ou intervenção; Reflexôes relativas ao ensino da terapia ocupacional na ABS; Discussões inseridas no campo de intervenção territorial e contextos comunitários cuja população alvo fosse definida por sua problemática de saúde/doença e/ ou processos de reabilitação; Pesquisas destinadas à análise de políticas públicas de saúde nesse contexto.

\section{Resultados}

\subsection{A produção bibliográfica nos periódicos da área}

Nos anos 2000 vimos emergir reflexóes ascendentes da categoria profissional sobre a atuação do terapeuta ocupacional nos serviços comunitários e territoriais no campo da saúde, incluindo as unidades da ABS. Tal afirmação é decorrente do resultado do levantamento realizado, no qual foram encontrados 21 artigos completos sobre $\mathrm{ABS}$ nas revistas científicas específicas de terapia ocupacional no Brasil, sendo 17 artigos na Revista de Terapia Ocupacional da USP e 4 nos Cadernos de Terapia Ocupacional da UFSCar.
A análise dos periódicos apresenta as discussóes dessa temática focada no estado de Sáo Paulo. Esse dado reflete a inserção da área de terapia ocupacional majoritariamente no contexto paulista, tanto em termos de número de profissionais, para a execução dos serviços, quanto de pesquisadores desenvolvendo conhecimento no campo. A presença de um percentual considerável de profissionais em Sáo Paulo, bem como a predominância e tradição de universidades com pesquisadores terapeutas ocupacionais no estado, trazem, como consequência, uma produção localizada do conhecimento na área.

No início dos anos 2000, os artigos de ambos periódicos remeteram para debates da categoria nas Unidades Básicas de Saúde (UBS). No período de 2001 a 2009, os Cadernos de Terapia Ocupacional da UFSCar não publicaram artigos na temática, sendo que o tema voltou a ser enfocado a partir de 2010, com 2 publicaçóes, e em 2011, com 1. Na Revista de Terapia Ocupacional da USP, a partir de 2002, as reflexóes iniciaram-se no âmbito do Programa de Saúde da Família, por meio da divulgaçáo de experiências de ensino e pesquisa desenvolvidas na Universidade de Sáo Paulo (USP) no espaço do PSF/QUALIS-SP ${ }^{3}$. Nota-se que, de 2005 a 2007, o periódico não publicou trabalhos na temática, que volta a ter destaque a partir de 2008.

A partir da análise da produção teórica acerca da atuação da terapia ocupacional na ABS, observa-se que dentre os 242 artigos publicados na Revista de Terapia Ocupacional da USP, 17 estão inseridos no campo em estudo, ou seja, $7 \%$ das publicaçóes. Já nos Cadernos de Terapia Ocupacional da UFSCar foram encontrados 4 artigos de sua publicação, que soma 161 textos, e que representam, aproximadamente, $2,5 \%$ dos artigos.

Relato de experiências acerca das açốes voltadas às pessoas com deficiência na ABS foi a temática mais encontrada: 12 trabalhos. As discussóes demonstram os caminhos para inserção da profissão nas açóes de reabilitaçáo na Atenção Básica em Saúde:

A bibliografia narra um esforço por parte de muitos profissionais em afirmar a inserção da profissão nas açóes de reabilitação na APS, a partir de um deslocamento epistemológico de bases organicistas para intervençóes dirigidas a aspectos socioparticipativos, independência e autonomia de pessoas e de grupos e constituiçáo de redes sociais de apoio [...] (ROCHA; SOUZA, 2011, p. 37).

$\mathrm{O}$ acesso às ações de reabilitação também foi um tema enfocado pelos terapeutas ocupacionais nos artigos pesquisados. Souza e Rocha (2010) identificaram que mesmo com as estratégias utilizadas pela equipe de reabilitação para a inclusão das 
Quadro 1. Relação dos artigos encontrados.

\section{Cadernos de Terapia Ocupacional da UFSCar - 2000-2011}

LOPES, R. E. Terapia Ocupacional em São Paulo, um percurso singular e geral. Cadernos de Terapia Ocupacional da UFSCar, São Carlos, v. 12, n. 2, p. 75-88, 2004.

BEIRÃO, R. O. S.; ALVES, C. K. A. Terapia Ocupacional no SUS: Refletindo sobre a normatização vigente. Cadernos de Terapia Ocupacional da UFSCar, São Carlos, v. 18, n. 3, p. 231-246, set./dez. 2010.

DUARTE, G. P.; UCHÔA-FIGUEIREDO, L. R. A vida cotidiana e a qualidade de vida de pacientes atendidos na atenção primária de saúde. Cadernos de Terapia Ocupacional da UFSCar, São Carlos, v. 18, n. 1, p. 19-33, 2010.

RODRIGUES, C. P. G. et al. Um olhar para a comunidade: experiência necessária para a formação do Terapeuta Ocupacional. Cadernos de Terapia Ocupacional da UFSCar, São Carlos, v. 19, n. 3, p. 343-350, 2011. http://dx.doi.org/10.4322/cto.2011.007

\section{Revista de Terapia Ocupacional da USP - 2000 ao primeiro volume de 2011}

TOLDRÁ, R. C.; PÉREZ, M. A. G.; MATTA, M. A. P. Acesso e qualidade da atenção à pessoa portadora de deficiência física em serviços públicos de saúde do município de Campinas. Revista de Terapia Ocupacional da USP, São Paulo, v. 11, n. 2-3, p. 72-8, maio/dez. 2000.

ALMEIDA, M. C.; TISSI, M. C.; OLIVER, F. C. Deficiências e atenção primária em saúde do conhecimento à invenção. Revista de Terapia Ocupacional da USP, São Paulo, v. 11, n.1, p. 33-42, jan./abr. 2000.

OLIVER, F. C. et al. Reabilitação no território: construindo a participação na vida social. Revista de Terapia Ocupacional da USP, São Paulo, v. 12, n. 1-3, p. 15-22, jan./dez. 2001.

LOPES, R. E. Políticas de saúde no Brasil: construções, contradições e avanços. Revista de Terapia Ocupacional da USP, São Paulo, v. 12, n. 1-3, p. 23-33, jan./dez. 2001.

ROCHA, E. F.; SHIMIZU, P. N.; BARRALES, L. M. Estágio de terapia ocupacional no programa da saúde da família: reflexões sobre uma parceria didático-assistencial entre o REATA/USP e o PSF/QUALIS-SP. Revista de Terapia Ocupacional da USP, São Paulo, v. 13, n. 3, p. 104-10, set./dez. 2002.

SIEGMAN, C.; PINHEIRO, C. A.; ALMEIDA, M. C. Terapia ocupacional e pacientes acamados: ações comunitárias. Revista de Terapia Ocupacional da USP, São Paulo, v. 13, n. 1, p. 37-43, jan./abr. 2002.

ALMEIDA, M. C.; CAMPOS, G. W. S. Políticas e modelos assistenciais em saúde e reabilitação de pessoas com deficiência no Brasil: análise de proposições desenvolvidas nas últimas duas décadas. Revista de Terapia Ocupacional da USP, São Paulo, v. 13, n. 3, p. 118-26, set./dez. 2002.

MALFITANO, A. P. S.; LOPES, R. E. Programa de saúde da família e agentes comunitários: demandas para além da saúde básica. Revista de Terapia Ocupacional da USP, São Paulo, v. 14, n. 3, p. 110-7, set./dez. 2003.

OLIVER, F. C. et al. Reabilitação com ênfase no território - Jardim D’Abril e Jardim Boa Vista, no município de São Paulo. Revista de Terapia Ocupacional da USP, São Paulo, v. 14, n. 3, p. 141-6, set./dez. 2003.

ROCHA, E. F.; PAULA, A. R.; KRETZER, M. R. O estudo de prevalência de deficiências e incapacidades como instrumento de planejamento das atividades de atenção à saúde e reabilitação no Programa Saúde da Família. Revista de Terapia Ocupacional da USP, São Paulo, v. 15, n. 1, p. 1-10, jan./abr. 2004.

JARDIM, T. A.; AFONSO, V. C.; PIRES, I. C. A terapia ocupacional na Estratégia de Saúde da Família evidências de um estudo de caso no município de São Paulo. Revista de Terapia Ocupacional da USP, São Paulo, v. 19, n. 3, p. 167-175, dez. 2008.

ROCHA, E. F.; KRETZER, M. R. Ações de reabilitação de pessoas com deficiência na estratégia da saúde da família da Fundação Zerbini e Secretaria Municipal de Saúde de São Paulo - Região Sudeste - Sapopemba/Vila Prudente - período 2000/2006. Revista de Terapia Ocupacional da USP, São Paulo, v. 20, n. 1, p. 59-67, jan./ abr. 2009.

RAFACHO, M.; OLIVER, F. C. A atenção aos cuidadores informais/familiares e a estratégia de Saúde da Família: contribuições de uma revisão bibliográfica. Revista de Terapia Ocupacional da USP, São Paulo, São Paulo, v. 21, n. 1, p. 41-50, 2010.

SOUZA, C. C. B. X.; ROCHA, E. F. Portas de entrada ou portas fechadas?: O acesso à reabilitação nas Unidades Básicas de Saúde da região sudeste do município de São Paulo - período de 2000 a 2006. Revista de Terapia Ocupacional da USP, São Paulo, São Paulo, v. 21, n. 3, p. 230-239, 2010.

ROCHA, E. F.; SOUZA, C. C. B. X. Terapia ocupacional em reabilitação na atenção primária à saúde: possibilidades e desafios. Revista de Terapia Ocupacional da USP, São Paulo, São Paulo, v. 22, n. 1, p. 36-44, 2011.

PIMENTEL, A. M.; COSTA, M. T. B.; SOUZA, F. R. Terapia Ocupacional na Atenção Básica: a construção de uma prática. Revista de Terapia Ocupacional da USP, São Paulo, São Paulo, v. 22, n. 2, p. 110-116, 2011.

MALFITANO, A. P. S.; FERREIRA, A. P. Saúde pública e terapia ocupacional: apontamentos sobre relações históricas e atuais. Revista de Terapia Ocupacional da USP, São Paulo, São Paulo, v. 22, n. 2, p. 102-109, 2011. 
demandas das pessoas com deficiência na ABS, a temática necessita ser mais discutida pelos usuários, profissionais de saúde, serviços, políticas públicas e órgãos governamentais, para que seja construído de forma efetiva e universal. Tal afirmação nos permite inferir que as pesquisas realizadas sobre essa temática não significam a incorporação das demandas das pessoas com deficiência na agenda política da ABS.

Acreditamos que a maioria das discussóes e publicaçôes com enfoque nas pessoas com deficiência pode relacionar-se com o maior número de grupos de pesquisa em terapia ocupacional voltados para o estudo e formação de profissionais para atuação junto a essa população. Lopes et al. (2010) publicaram um estudo sobre o levantamento de grupos de pesquisa cadastrados no Diretório de Grupos de Pesquisa do CNPq (na grande área da Saúde, subárea de Fisioterapia e Terapia Ocupacional com a expressão Terapia Ocupacional no nome do grupo, no título de linha de pesquisa ou em suas palavras-chave) relatando a presença de 30 grupos registrados, sendo que 26 eram coordenados por terapeutas ocupacionais e estavam ativos, com $84 \%$ deles localizados na região Sudeste do Brasil. Dentre esses grupos estudados identificamos pelo menos 7 voltados às temáticas relacionadas às pessoas com deficiência e 2 voltados especificamente para a área de saúde mental.

Não encontramos artigos específicos sobre a atuação do terapeuta ocupacional na ABS junto às pessoas com sofrimento mental, entretanto localizamos artigos voltados às açóes na saúde mental em serviços extra-hospitalares - especialmente nos Centros de Atenção Psicossocial (CAPS) e em açôes territoriais comunitárias em saúde mental (NICÁCIO; CAMPOS, 2005; MÂNGIA; MURAMOTO, 2006), que dialogam com a atuação do terapeuta ocupacional na Atenção Básica em
Saúde no contexto de cuidado às pessoas com sofrimento mental.

Compondo as discussóes da categoria na ABS, também encontramos 1 artigo que fez referência às propostas voltadas às pessoas com diabetes $\mathrm{e}$ hipertensão, de Duarte e Uchôa-Figueiredo (2010), o qual aborda a investigaçáo da qualidade de vida e do cotidiano dos pacientes que frequentavam o grupo de prevenção do programa de aprimoramento multiprofissional em hipertensão arterial sistêmica e diabetes mellitus do município de Ribeirão Preto, SP.

Segundo as autoras, a estratégia de grupos visando à promoção de saúde tem contribuído para que as pessoas consigam se adaptar às mudanças decorrentes da doença e, também, possibilita-lhes a construção de um envelhecimento saudável. Nessa perspectiva, segundo as autoras, toda a equipe de saúde na $A B S$, inclusive o terapeuta ocupacional, deve prestar sua contribuiçáo no controle desses fatores, voltado para a modificação de hábitos, rotinas e papéis ocupacionais.

Os demais artigos, em número de 8 , problematizavam a política prevista pelo SUS. Nessa direção, Beirão e Alves (2011) apresentaram a inserção e as áreas de atuação da terapia ocupacional no SUS a partir do levantamento das portarias do Ministério da Saúde nos últimos 10 anos. As autoras encontraram 41 portarias vigentes que fazem referência ao terapeuta ocupacional, cuja maioria se concentra na área de reabilitação. Também foram estudados os procedimentos previstos no SUS para a categoria, que se relacionam, em sua maioria, à atuação dos profissionais nos Centros de Atenção Psicossocial, demonstrando, segundo os autores, que a profissão já se encontra inserida e bem sistematizada na saúde mental do SUS.

Tal dado é passível de discussão, na medida em que o campo da saúde mental questiona os

Quadro 2. Formatação dos anais dos congressos brasileiros de Terapia Ocupacional.

\begin{tabular}{|c|c|c|c|c|c|}
\hline $\begin{array}{c}\text { Congresso } \\
\text { Brasileiro de Terapia } \\
\text { Ocupacional }\end{array}$ & $\begin{array}{c}\text { Indicações } \\
\text { dos autores }\end{array}$ & $\begin{array}{c}\text { Formato dos } \\
\text { anais }\end{array}$ & $\begin{array}{c}\text { Distinção entre } \\
\text { modalidades de } \\
\text { apresentação }\end{array}$ & Resumos & $\begin{array}{c}\text { Trabalhos } \\
\text { completos }\end{array}$ \\
\hline VI CBTO - 1999 & Sim & Papel & Sim & Sim & Não \\
\hline VII CBTO - 2001 & Sim & Papel & Sim & Sim & Não \\
\hline VIII CBTO - 2003 & Não & Papel & Sim & Não & Não \\
\hline IX CBTO - 2005 & Sim & CD & Não & Sim & Sim \\
\hline X CBTO - 2007 & Sim & CD & Sim & Sim & Sim \\
\hline XI CBTO - 2009 & Sim & CD & Sim & Sim & Não \\
\hline XII CBTO - 2011 & Sim & Meio eletrônico & Sim & Sim & Sim \\
\hline
\end{tabular}

Fonte: Anais dos VI, VII, VIII, IX, X, XI e XII CBTOs. (CONGRESSO..., 1999, 2001, 2003, 2005, 2007, 2009, 2011). 
limites de inserção da política da área, assim como, especificamente, da participação do terapeuta ocupacional. O levantamento apontado das portarias ministeriais reflete a instituição formal da categoria nas normatizações da área, sendo mais expressivo do que naquelas que se relacionam às pessoas com deficiência, que são, por si, em menor volume, demonstrando menor inserção daquele grupo populacional na agenda política.

Com os dados levantados nos artigos analisados, observa-se, ainda, uma produção incipiente em artigos científicos, fator presente na Terapia Ocupacional como um todo, sendo que a produção encontrada centra-se na divulgação de conhecimento de pesquisadores da área traduzindo, parcialmente, o que a prática profissional tem constituído nesse campo.

\subsection{A produção em congressos brasileiros}

Nas produções apresentadas nos congressos brasileiros de Terapia Ocupacional, nota-se uma ascendência numérica nas discussóes da categoria sobre essa temática no período de 1999 a 2011 (Figura 1, Quadro 3), sendo que os congressos agregam um expressivo número de docentes, discentes e profissionais da área.

Entretanto, a partir de 2005, no IX CBTO, há uma estabilidade no percentual de trabalhos apresentados, ocupando cerca de 5\% das discussóes dessa natureza. Tendo em vista o aumento do número de profissionais na Atenção Básica em Saúde (CALDEIRA, 2009), pode-se dizer que suas reflexôes ainda não foram traduzidas em trabalhos apresentados no evento científico da categoria, na medida em que se manteve o percentual na área.

O VI CBTO aconteceu em 1999, na cidade de Águas de Lindóia, SP, quando tivemos 227 trabalhos (não incluso a modalidade de vídeos na análise). De acordo com a nossa seleção, encontramos 6 trabalhos na temática pesquisada. No VII CBTO, ocorrido em 2001 na cidade de Porto Alegre, RS, em que foram apresentados 394 trabalhos, encontramos 16 trabalhos na temática. No CBTO seguinte, tivemos dados destoantes do crescimento que vinha sendo observado sobre a temática: no VIII CBTO, que ocorreu na

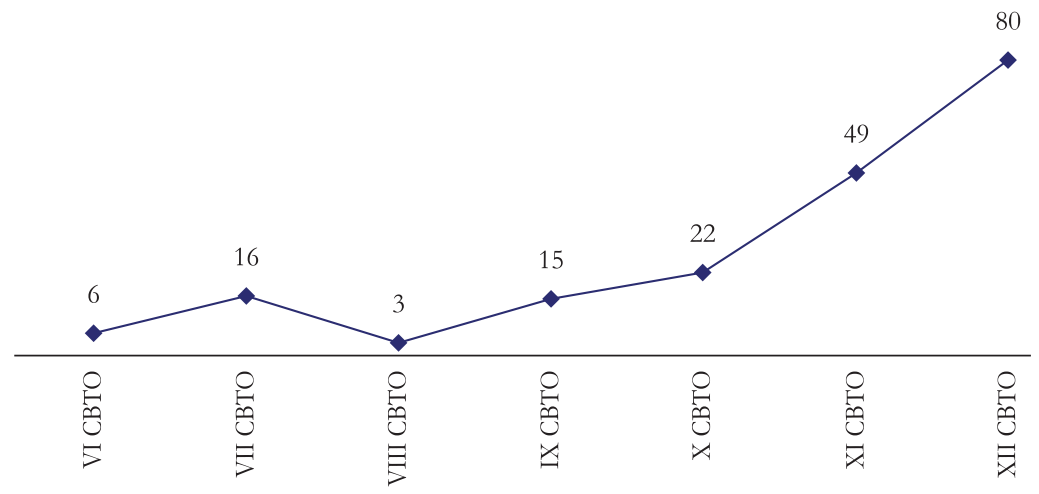

Fonte: ANAIS do VI CBTO, VII CBTO, VIII CBTO, IX CBTO, X CBTO, XI CBTO e XII CBTO.

Figura 1. Número de trabalhos selecionados nos CBTOs 1999-2011.

Quadro 3. Número de trabalhos selecionados na temática.

\begin{tabular}{|c|c|c|c|}
\hline $\begin{array}{c}\text { Congresso Brasileiro de } \\
\text { Terapia Ocupacional }\end{array}$ & $\begin{array}{c}\text { Número de trabalhos } \\
\text { apresentados }\end{array}$ & $\begin{array}{c}\text { Número de trabalhos } \\
\text { selecionados }\end{array}$ & $\begin{array}{c}\text { Porcentagem } \\
\text { (\%) }\end{array}$ \\
\hline VI CBTO & 227 & 6 & 2,6 \\
\hline VII CBTO & 394 & 16 & 4,0 \\
\hline VIII CBTO & 261 & 3 & 1,1 \\
\hline IX CBTO & 286 & 15 & 5,2 \\
\hline X CBTO & 472 & 22 & 4,6 \\
\hline XI CBTO & 834 & 49 & 5,8 \\
\hline XII CBTO & 1.281 & 80 & 6,2 \\
\hline Total & 3.755 & 191 & 5,0 \\
\hline
\end{tabular}

Fonte: Anais do VI, VII, VIII, IX, X, XI e XII CBTOs. (CONGRESSO..., 1999, 2001, 2003, 2005, 2007, 2009, 2011). 
cidade de Foz do Iguaçu, PR, em 2003, foram menos trabalhos apresentados e náo foi publicado anais com resumos. Tivemos acesso apenas aos títulos dos trabalhos apresentados, material disponibilizado pela organização do congresso aos participantes: foram 261 trabalhos no total, sendo que 3 se adequaram à nossa seleçấo. Esse congresso teve uma diminuição significativa de trabalhos apresentados, em seu todo, devido a questóes relacionadas à organização e estruturação do evento.

No ano de 2005 aconteceu o IX CBTO, em Recife, PE. Dos 286 trabalhos publicados, selecionamos 16 , porém excluímos 1, cujo arquivo não foi disponibilizado para análise, totalizando 15 trabalhos. Em 2007 aconteceu o X CBTO, na cidade de Goiânia, GO), com 472 trabalhos apresentados no total, dos quais 22 inseriam-se na temática em estudo. Em 2009, no XI CBTO, que aconteceu em Fortaleza, CE, encontramos 49 trabalhos na temática em um total de 834 trabalhos apresentados. E, por fim, destacamos que em 2011, no XII CBTO e IX Congresso Latino Americano de Terapia Ocupacional, realizado em São Paulo, SP, foram apresentados 1.281 trabalhos, sendo que 80 deles, ou seja, 6,2\%, referiam-se à Atenção Básica em Saúde, conforme demonstra a Figura 2.

Esse panorama demonstra que o tema, ao longo dos eventos estudados, foi ganhando espaço nas mesas e conferências, sendo que no último encontro observou-se a maior expressão da temática: durante ele ocorreu um minicurso específico, enfocando a atuação da profissão junto ao NASF, e o I Simpósio de Terapia Ocupacional em Atenção Primária em Saúde.
De acordo com os anais, o minicurso buscou divulgar e promover a importância do trabalho em equipe; apoiar e incentivar a implementação de atividades compartilhadas; fortalecer o trabalho do terapeuta ocupacional através de alinhamento conceitual sobre APS, NASF e Apoio Matricial; discutir competências e atribuiçôes do terapeuta ocupacional no NASF.

Objetivando promover reflexões sobre a atuação e os desafios atuais para a consolidaçáo da Terapia Ocupacional na Atenção Primária em Saúde no contexto do SUS, assim como possibilitar a articulação de um coletivo de profissionais comprometidos com o desenvolvimento da profissão nesse campo de assistência, de ensino e de pesquisa, o I Simpósio de Terapia Ocupacional em Atenção Primária em Saúde apresentou os seguintes grupos de estudos: Formação do terapeuta ocupacional para o trabalho na APS; Trabalho em equipe e matriciamento na APS; Terapia Ocupacional na atenção primária: atribuiçóes e tecnologias; População atendida em terapia ocupacional na APS.

Considerando os CBTOs enfocados, quanto aos trabalhos apresentados, dos 191 selecionados (Figura 3), 41\% deles enfocavam a inserção da profissão na Atenção Básica em Saúde sem definir a população alvo das açóes. Os trabalhos discutiam o campo de atuação do profissional, inicialmente nas Unidades Básicas de Saúde, posteriormente nas Unidades de Saúde da Família (USF). Relatavam, ainda, aspectos sobre a atuação do terapeuta ocupacional na ABS. Por exemplo, o trabalho de Marque et al. (2009) apresenta as intervençóes do profissional a partir de ações na comunidade, com

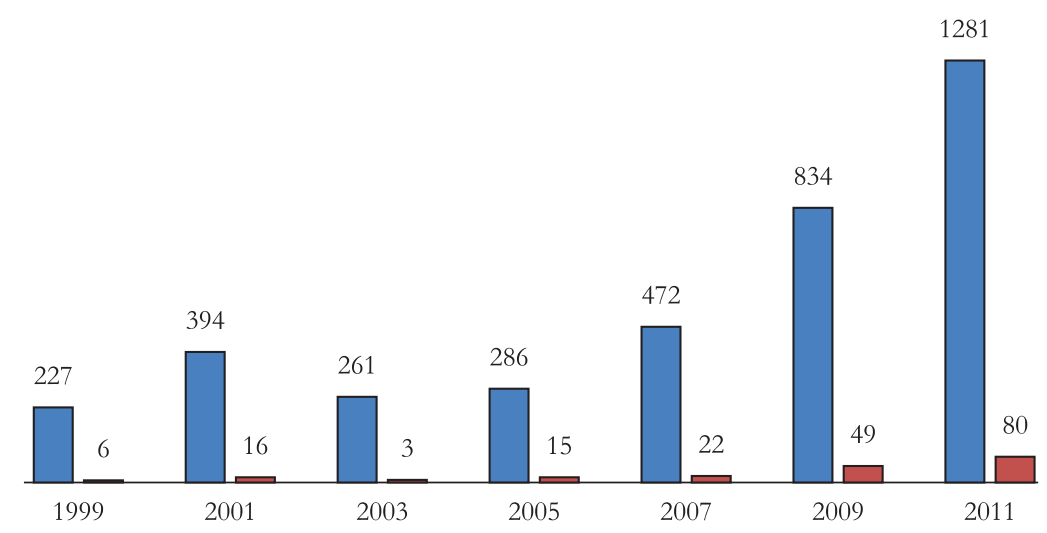

Fonte: ANAIS do VI CBTO, VII CBTO, VIII CBTO, IX CBTO, X CBTO, XI CBTO e XII CBTO.

Figura 2. Número de trabalhos selecionados nos CBTO 1999-2011. Fonte: anais do VI, VII, VIII, IX, $\mathrm{X}, \mathrm{XI}$ e XII CBTOs. 
usuários nas USF e UBS e com as equipes de saúde. Autores como Sampaio e Godinho (2009) referem que o campo da APS permite dar visibilidade à profissão, tanto perante a comunidade atendida quanto as demais profissionais, assim como possibilita ao terapeuta ocupacional lidar com o cotidiano dos usuários, dentro de uma proposta de intervenção voltada à promoção da saúde no território.

Para Nunes (2009), o terapeuta ocupacional privilegia-se desse campo de atuação por considerar os múltiplos aspectos e contextos de vida para sua intervenção, inserindo-se na ESF por meio do desenvolvimento de açóes na comunidade, na residência das pessoas e, em especial, em espaços comunitários e sociais, ampliando o serviço de saúde para além de seus limites físicos institucionais.

Desde 2001 são apresentados trabalhos voltados às práticas de ensino da terapia ocupacional na Atenção Básica em Saúde (ROCHA; SHIMIZO, 2001). Segundo Souza e Rocha (2009), o processo ensino-aprendizagem, no contexto da APS, contribui para a interaçáo dos alunos e dos usuários com a comunidade, incluindo espaços da sociedade civil organizada, como os movimentos sociais. A utilização de tais espaços para a formação profissional facilita a compreensão sobre o SUS e suas articulaçôes necessárias, levando em consideração, para além do diagnóstico, o território e a comunidade como lócus de intervenção.

A partir do X CBTO, que ocorreu em 2007, os debates incluem as atividades de formação profissional na graduação e pós-graduação, com enfoque para o Programa de Educação Tutorial para o Trabalho em Saúde (PET-SAÚDE) e o Programa de Residência Multiprofissional, entendidas, pelos autores, como estratégias de articulação entre os cursos de graduação em saúde e os serviços da APS, sendo especialmente potentes para a formação profissional voltada às

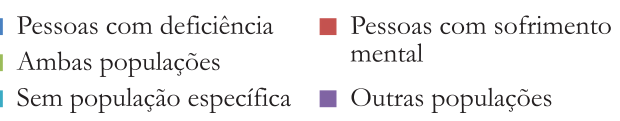

Pessoas com sofrimento mental

Outras populações

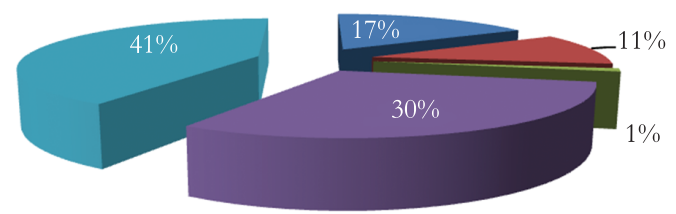

Figura 3. Populações alvo das práticas dos terapeutas ocupacionais nos trabalhos apresentados nos CBTOs de 1999 a 2011. Fonte: Anais do VI, VII, VIII, IX, X, XI e XII CBTOs. necessidades do SUS (SOARES; OLIVEIRA, 2007; ARRUDA; MOREIRA, 2009).

Em 2009, no XI CBTO, emergem as discussōes da profissão junto aos Núcleos de Apoio à Saúde da Família (NASF), notadamente aquelas vinculadas às açôes previstas na Portaria GM n. 154, de 24 de janeiro de 2008, que institui o terapeuta ocupacional como profissional que pode compor as equipes de Reabilitação e Saúde Mental do NASF.

Segundo Pinho e Almeida (2011), não se trata apenas de garantir por lei a inserção do terapeuta ocupacional nas políticas públicas, por meio das açôes no NASF, mas de definir o perfil das atividades a serem desenvolvidas por esse profissional nas políticas públicas.

Nesse contexto, os terapeutas ocupacionais devem seguir lutando para que integrem de fato o conjunto de profissionais que atuam no Sistema Único de Saúde (SUS). Aparentemente, não apenas estar incluído no NASF será o instrumento que proporcione essa inserção [...] (PINHO; ALMEIDA, 2011).

Prosseguindo com nossa análise, as pessoas com deficiência física foram alvo de $17 \%$ dos trabalhos selecionados. Grande parte deles enfocou açóes com participação comunitária na cidade de São Paulo e intervençóes profissionais voltadas para as necessidades coletivas de grupos populacionais como pessoas com deficiência, além de apresentar a Reabilitação Baseada na Comunidade (RBC) como estratégia para atuação do terapeuta ocupacional na Atenção Primária em Saúde (OLIVER; AOKI; TISSI, 1999; OLIVER; NICOLAU; TISSI, 2005; OLIVER; AOKI; NICOLAU, 2007; CALDEIRA; OLIVER, 2009), conforme Figura 3.

Aproximadamente $11 \%$ dos trabalhos enfocaram os desafios e as açóes junto às pessoas com sofrimento mental na ABS. Segundo Brito (2009), as pessoas com sofrimento mental e suas redes de apoio necessitam ser atendidas em suas reais necessidades e, para tal, todas as equipes de saúde precisam estar organizadas. Dessa maneira faz-se necessário repensar a assistência em saúde mental, suas implicaçóes e articulaçóes para o desenvolvimento de açôes dentro da rede básica em saúde.

Destacamos, também, que alguns trabalhos relataram a atuação do terapeuta ocupacional na saúde mental através das equipes de retaguarda ou apoio em saúde mental, agindo como instrumento facilitador da articulação das redes de suporte e para a inclusão na comunidade (SALES, 2005). 
Muito se discute sobre as experiências de prevenção e promoção de saúde junto à APS, entretanto, também encontramos o relato de cuidado interdisciplinar prestado às pessoas com diagnóstico de esquizofrenia na APS (HOLANDA; MENEZES, 2009), demonstrando que os cuidados na rede básica de saúde podem incluir todas as populaçóes daquele território e açóes preventivas e de reabilitaçáo.

Nesse enfoque, aproximadamente $1 \%$ dos trabalhos destacaram conjuntamente as pessoas com deficiência e as pessoas com sofrimento mental, visando discutir a inclusão na ESF de demandas comunitárias e daquelas referentes às problemáticas não tradicionalmente acolhidas pelos serviços de saúde, procurando compreender como as políticas de saúde podem se tornar instrumentos de inclusão ou exclusão de grupos populacionais ou problemáticas náo acolhidas, criando um padrão de proteção social diferenciado que intervenha diretamente na vida da população assistida (LOPES; SOUZA; PALMA, 2005; LOPES; MALFITANO; PALMA, 2005; CARRASCO-BASSI; MALFITANO, 2011).

Os trabalhos que se referiam às açôes dos terapeutas ocupacionais na APS com outras populaçôes (que não as tradicionalmente enfocadas pela categoria: as pessoas com deficiência e as pessoas com sofrimento mental), somaram $30 \%$ - entre elas destacamos: idosos, hipertensos e diabéticos, crianças de 0 a 3 anos, crianças em idade escolar, cuidadores, pessoas obesas, gestantes e puérperas, mães adolescentes, jovens e adolescentes, entre outros.

De uma maneira geral, os trabalhos demonstram que os terapeutas ocupacionais brasileiros desenvolvem práticas com todas as populaçôes cuidadas pela ABS, inclusive aquelas com maior prevalência de doenças e com programas estabelecidos para intervenção.

Mesmo frente a esse panorama, que certamente contribui para o crescimento da Terapia Ocupacional, partilhamos de uma concepção que atribui importância à atuação do terapeuta ocupacional frente às necessidades das pessoas com sofrimento mental e das pessoas com deficiência, populaçóes clássicas de estudo e intervenção desses profissionais e, em sua maioria, não acolhidas tradicionalmente pela Atenção Básica em Saúde. O terapeuta ocupacional pode ser um mediador para inclusão das demandas dessas populaçôes junto às equipes de saúde e auxiliar no estabelecimento de redes sociais de suporte junto ao território, na perspectiva de uma oferta de saúde integral para todos.

Os terapeutas ocupacionais vinculados à atençâo básica e territorial em saúde tendem a contribuir para tornar a atenção mais completa e contextualizada pela natureza dos problemas que têm buscado trabalhar junto a pessoas e/ ou grupos, como também têm possibilidade de serem articuladores de alternativas para que as pessoas que acompanham acessem outros níveis da assistência [...] (ALMEIDA; OLIVER, 2001, p. 11).

\section{Considerações finais}

O levantamento apresentado demonstrou ser crescente a divulgação de conhecimento e de experiências acerca da Terapia Ocupacional na ABS. Contudo, nota-se que o campo da ABS para o terapeuta ocupacional possui ainda uma literatura incipiente, sendo, entretanto, um resultado previamente esperado a esse levantamento, na medida em que a intervenção no nível primário de saúde vem acumulando recentemente pesquisas, intervençôes e desenvolvimento específicos. Desse modo, observamos uma ascendência das discussóes na categoria sobre essa temática, na última década.

Em nossa pesquisa, nos artigos completos publicados na temática em revistas de Terapia Ocupacional, vimos que a maioria se refere à atuaçáo do terapeuta ocupacional junto às pessoas com deficiência na $\mathrm{ABS}$ e estão, principalmente, ligados às ações de pesquisa e de ensino realizadas em universidades paulistas.

Já os trabalhos apresentados nos CBTOs apresentam algumas diferenças de resultado, uma vez que englobam um escopo maior de autores, pela sua abrangência. A análise de tais trabalhos, de uma maneira geral, confirmou que os terapeutas ocupacionais brasileiros estâo desenvolvendo práticas com todas as populaçóes cuidadas pela ABS, inclusive aquelas que não são, tradicionalmente, alvo de intervenção de terapeutas ocupacionais e que apresentam doenças com maior prevalência na população em geral. Programas estabelecidos e com incentivo para sua implantaçáo, como aqueles dirigidos a hipertensos e diabéticos, gestantes e idosos, têm contado com a participação de terapeutas ocupacionais na sua operacionalizaçáo.

Ao propor ações de promoção, proteção e recuperação da saúde dos indivíduos, grupos e comunidades, do recém-nascido ao idoso, sadios ou doentes, de forma integral e contínua, a ABS responsabiliza-se pelo cuidado de saúde de todos, inclusive das pessoas com deficiência e das pessoas com sofrimento mental. Entretanto, pesquisas recentes (MALFITANO, 2007; FIGUEIREDO; ONOCKO CAMPOS, 2009; SOUZA; ROCHA, 
2011) demostraram que as demandas que ultrapassam o âmbito da saúde básica (entendida aqui como os cuidados às doenças de maior prevalência) são, muitas vezes, encaminhadas para outros serviços, recebendo pouca assistência da Atenção Básica em Saúde.

Nessa direção, buscando trazer o foco para a população com deficiência e sofrimento mental, preocupação de grande relevância para o terapeuta ocupacional, fica assinalada a necessidade de melhor compreender o tipo de assistência que tem sido ofertada no âmbito da ABS para esses grupos. Tal preocupação fica evidenciada para o terapeuta ocupacional uma vez que também direciona sua atuação para a autonomia e a inserção social de tais pessoas. Parte-se da necessidade da corresponsabilização efetiva das equipes de saúde da ABS para as demandas de todos pois, ao tratar das questóes de saúde, faz-se necessário buscar as articulaçóes possíveis no que tange à noção de valor da vida e da saúde.

Especificamente com relação às pessoas com deficiência e sofrimento mental, fazem-se necessárias intervençôes que permitam que a ação de saúde não seja apenas pontual, no sentido de avaliar um sofrimento agudo, mas, principalmente, uma ação contínua, de cuidado e de oferta de suporte às condiçôes de vida (CARNEIRO JUNIOR; SILVEIRA, 2003).

Por fim, vale destacar que os investimentos governamentais na Atenção Básica em Saúde têm promovido uma maior inserção e visibilidade do terapeuta ocupacional nesse escopo de atuaçáo, trazendo, como consequência, uma ascendência nas discussóes acerca de suas intervençóes. Portanto, conclui-se que o tema vem sendo aprimorado no interior da profissão, ainda requerendo um aprofundamento de pesquisas que se dediquem à produção de conhecimento nesse campo, com o intuito de acurar a aplicação de tecnologias sociais voltadas ao acesso à saúde e melhora de condições de vida de diversos grupos populacionais que utilizam os serviços de Terapia Ocupacional.

\section{Referências}

AlMEIDA, M. C.; OLIVER, F. C. Abordagens Comunitárias e territoriais em reabilitação de pessoas com deficiências: fundamentos para a Terapia Ocupacional. In: CARLO, M. M. R. P.; BARTALOTTI, C. C. (Orgs.). Terapia Ocupacional no Brasil: fundamentos e perspectivas. Sáo Paulo: Plexus, 2001. v. 1, p. 81-98.

ARRUDA, A. E.; MOREIRA, P. T. PET-SAÜDE uma estratégia de articulação entre a TO e os serviços de atenção primária à saúde. In: CONGRESSO BRASILEIRO DE
TERAPIA OCUPACIONAL, 11., 2009, Fortaleza. Anais... Fortaleza: Associação dos Terapeutas Ocupacionais do Ceará, 2009. CD-ROM.

CARRASCO-BASSI, B. G.; MALFITANO, A. P. S. Políticas Públicas, Terapia Ocupacional e Atenção Básica em Saúde: Caminhos da Assistência às Pessoas com deficiência e Sofrimento mental no município de São Carlos, SP. In: CONGRESSO BRASILEIRO DE TERAPIA OCUPACIONAL, 12., 2011, São Paulo. Anais... São Paulo: Associação dos Terapeutas Ocupacionais do Estado de São Paulo, 2011. CD-ROM.

BEIRÃO, R. O. S.; ALVES, C. K. A. Terapia Ocupacional no SUS: refletindo sobre a normatização vigente. Cadernos de Terapia Ocupacional da UFSCar, Sáo Carlos, v. 18, n. 3, p. 231-246, 2010.

BRASIL. Ministério da Saúde. Departamento de Atenção Básica. Política Nacional de Atenção Básica. Brasília: Ministério da Saúde, 2006.

BRASIL. Ministério da Saúde. Departamento de Atenção Básica. Estratégia de Saúde da Família. Brasília: Ministério da Saúde, 2010a. Disponível em: <www.saude.gov.br>. Acesso em: 07 maio 2010.

BRASIL. Ministério da Saúde. Secretaria de Atenção à Saúde. Departamento de Atenção Básica. Diretrizes do NASF: Núcleo de Apoio à Saúde da Família. Brasília: Ministério da Saúde, 2010b.

BRITO, J. S. Terapia Ocupacional, uma prática possível para a atenção básica em saúde. In: CONGRESSO BRASILEIRO DE TERAPIA OCUPACIONAL, 11., 2009, Fortaleza. Anais... Fortaleza: Associação dos Terapeutas Ocupacionais do Ceará, 2009. CD-ROM.

CALDEIRA, V. A. Prática de Terapia Ocupacional em unidade básica de saúde na atenção às pessoas com deficiência. 2009. 170 f. Dissertação (Mestrado em Ciências)-Faculdade de Medicina da Universidade de São Paulo, São Paulo, 2009.

CAldeirA, V. A.; Oliver, F. C. Prática de terapia ocupacional na atenção básica em saúde. In: CONGRESSO BRASILEIRO DE TERAPIA OCUPACIONAL, 11., 2009, Fortaleza. Anais... Associação dos Terapeutas Ocupacionais do Ceará, 2009. CD-ROM.

CAMPOS, G. W. S. Modelos assistenciais e Unidades Básicas de Saúde: elementos para debate. In: MERHY, E. E. (Org.) Planejamento sem normas. Sáo Paulo: Hucitec, 1989. p. 53-60.

CARNEIRO JUNIOR, N.; SILVEIRA, C. Organização das práticas de atenção primária em saúde no contexto dos processos de exclusão/inclusão social. Cadernos de Saúde Pública, Rio de Janeiro, v. 19, n. 6, p. 1827-1835, 2003.

CASTRO, A. L. B. A condução federal da política de atenção primária à saúde no Brasil: continuidades e mudanças no período de 2003 a 2008. 2009. 215 f. Dissertação (Mestrado em Saúde Pública)-Escola Nacional de Saúde Pública, Fundação Oswaldo Cruz, Rio de Janeiro, 2009.

CONGRESSO BRASILEIRO DE TERAPIA OCUPACIONAL - CBTO, 6., 1999, Águas de Lindóia. 
Anais... São Paulo: Associação Paulista de Terapeutas Ocupacionais, 1999. $91 \mathrm{p}$.

CONGRESSO BRASILEIRO DE TERAPIA OCUPACIONAL - CBTO, 7., 2001, Porto Alegre. Anais... Porto Alegre: Associação dos Terapeutas Ocupacionais do Rio Grande do Sul, 2001. 208 p.

CONGRESSO BRASILEIRO DE TERAPIA OCUPACIONAL - CBTO, 8., 2003, Foz do Iguaçu. Anais... Foz do Iguaçu: Associação Paranaense de Terapeutas Ocupacionais, 2003. 17 p.

CONGRESSO BRASILEIRO DE TERAPIA OCUPACIONAL - CBTO, 9., 2005, Recife. Anais... Recife: Associação dos Terapeutas Ocupacionais de Pernambuco, 2005. CD-ROM.

CONGRESSO BRASILEIRO DE TERAPIA OCUPACIONAL - CBTO, 10., 2007, Goiânia. Anais... Goiânia: Associação dos Terapeutas Ocupacionais de Goiás, 2007. CD-ROM.

CONGRESSO BRASILEIRO DE TERAPIA OCUPACIONAL - CBTO, 11., 2009, Fortaleza. Anais... Fortaleza: Associação dos Terapeutas Ocupacionais do Ceará, 2009. CD-ROM.

CONGRESSO BRASILEIRO DE TERAPIA OCUPACIONAL - CBTO, 12., 2011, São Paulo. Anais... São Paulo: Associação dos Terapeutas Ocupacionais do Estado de São Paulo, 2011. CD-ROM.

DUARTE, G. P.; UCHÔA-FIGUEIREDO, L. R. A vida cotidiana e a qualidade de vida de pacientes atendidos na atenção primária de saúde. Cadernos de Terapia Ocupacional da UFSCar, São Carlos, v. 18, n. 1, p. 19-33, 2010.

FIGUEIREDO, M. D.; ONOCKO CAMPOS, R. Saúde Mental na atenção básica à saúde de Campinas, SP: uma rede ou um emaranhado? Ciência \& Saúde Coletiva, Rio de Janeiro, v. 14, n. 1, p. 129-138, 2009. http://dx.doi. org/10.1590/S1413-81232009000100018

HOLANDA, M. S. S.; MENEZES, K. Olhar interdisciplinar na visita domiciliar as pessoas com esquizofrenia: uma experiência da TO na ESF no município Sobral/CE. In: CONGRESSO BRASILEIRO DE TERAPIA OCUPACIONAL, 11., 2009, Fortaleza. Anais... Fortaleza: Associação dos Terapeutas Ocupacionais do Ceará, 2009. CD-ROM.

LOPES, R. E. et al. Pesquisa em terapia ocupacional: apontamentos acerca dos caminhos acadêmicos no cenário nacional. Revista Terapia Ocupacional da Universidade de São Paulo, São Paulo, v. 21, n. 3, p. 207-214, 2010.

LOPES, R. E.; MALFITANO, A. P. S.; PALMA, A. Agentes Comunitários de saúde e as demandas de saúde mental e de pessoas portadoras de deficiência no território. In: CONGRESSO BRASILEIRO DE TERAPIA OCUPACIONAL, 9., 2005, Recife. Anais... Recife: Associação dos Terapeutas Ocupacionais de Pernambuco, 2005. CD-ROM.

LOPES, R. E; SOUZA, R. G.; PALMA, A. M. Educação em saúde - Ampliando o conhecimento e atuação dos agentes comunitários de saúde no contexto da saúde mental. In: CONGRESSO BRASILEIRO DE
TERAPIA OCUPACIONAL, 9., 2005, Recife. Anais... Recife: Associação dos Terapeutas Ocupacionais de Pernambuco, 2005. CD-ROM.

MALFITANO, A. P. S.; LOPES, R. E. Programa de Saúde da Família e Agentes Comunitários: Demandas para além da saúde básica. Revista Terapia Ocupacional da Universidade de São Paulo, Sáo Paulo, v. 14, n. 3, p. 110-117. 2003.

MALFITANO, A. P. S. Atrás da porta que se abre: Demandas sociais e o Programa de Saúde da Família. Holambra: Editora Setembro, 2007.

MÂNGIA, E. F.; MURAMOTO, M. Integralidade e construção de novas profissionalidades no contexto dos serviços substitutivos de saúde mental. Revista Terapia Ocupacional da Universidade de São Paulo, São Paulo, v. 17, n. 3, p. 115-122, 2006.

MARQUE, B.; BARROS, A. C. B; BAIRLE, T. P. Programa de saúde da família. Uma nova possibilidade à terapia ocupacional? In: CONGRESSO BRASILEIRO DE TERAPIA OCUPACIONAL, 11., 2009, Fortaleza. Anais... Fortaleza: Associação dos Terapeutas Ocupacionais do Ceará, 2009. CD-ROM.

MOREIRA, A. B. Terapia Ocupacional: História Crítica e Abordagem Territorial e Comunitária. Vita et Sanitas, Trindade, v. 2, n. 2, p. 79-91, 2008.

NICÁCIO, F.; CAMPOS, G. W. S. C. Instituições de "portas abertas": novas relaçóes usuários-equipes-contextos na atenção em saúde mental de base comunitária/territorial. Revista Terapia Ocupacional da Universidade de São Paulo, Sáo Paulo, v. 16, n. 1, p. 40-46, 2005.

NUNES, E. F. S. Novas perspectivas no cotidiano do TO na rede básica de saúde. In: CONGRESSO BRASILEIRO DE TERAPIA OCUPACIONAL, 11., 2009, Fortaleza. Anais... Fortaleza: Associação dos Terapeutas Ocupacionais do Ceará, 2009. CD-ROM.

OLIVER, F. C.; AOKI, M.; TISSI, M. C. Reabilitação Baseada na Comunidade- Discutindo estratégias de ação no contexto sociocultural. In: CONGRESSO BRASILEIRO DE TERAPIA OCUPACIONAL, 6., 1999, Águas de Lindóia. Anais... São Paulo: Associação Paulista de Terapeutas Ocupacionais, 1999. $91 \mathrm{p}$.

OLIVER, F. C.; AOKI, M.; NICOLAU, S. M. Reabilitação baseada na comunidade e terapia ocupacional: desafios para a construção de um campo de conhecimento e de atenção. In: CONGRESSO BRASILEIRO DE TERAPIA OCUPACIONAL, 10., 2007, Goiânia. Anais... Goiânia Associação dos Terapeutas Ocupacionais de Goiás, 2007. CD-ROM.

OLIVER, F. C.; NICOLAU, S. M.; TISSI, M. C. Práticas territoriais, terapia ocupacional e pessoas com deficiência: Construindo um campo de intervenção. In: CONGRESSO BRASILEIRO DE TERAPIA OCUPACIONAL, 9., 2005, Recife. Anais... Recife: Associação dos Terapeutas Ocupacionais de Pernambuco, 2005. CD-ROM.

PINHO, P. A.; ALMEIDA, R. Z. Atuação do Terapeuta Ocupacional no Contexto NASF: Um olhar para a Atençáo Básica em Saúde. In: CONGRESSO BRASILEIRO DE TERAPIA OCUPACIONAL, 12., 2011, São Paulo. 
Anais... São Paulo: Associação dos Terapeutas Ocupacionais do Estado de São Paulo, 2011. CD-ROM.

ROCHA, E. P.; SHIMIZO, P. N. Implantação do Estágio de Atuação em T.O no programa Qualis/PSF do município de São Paulo. In: CONGRESSO BRASILEIRO DE TERAPIA OCUPACIONAL, 7., 2001, Porto Alegre. Anais... Porto Alegre: Associação dos Terapeutas Ocupacionais do Rio Grande do Sul, 2001. 208 p.

ROCHA, E. F.; SOUZA, C. C. B. X. Terapia Ocupacional em reabilitação na Atenção Primária à Saúde: possibilidades e desafios. Revista Terapia Ocupacional da Universidade de São Paulo, São Paulo, v. 22, n. 1, p. 36-44, 2011.

SALES, I. C. B. A importância das equipes de retaguarda em saúde mental como instrumento facilitador da articulaçáo de redes e suporte ao PSF para a (re) inclusão do ex-usuário de CAPS na sua comunidade. In: CONGRESSO BRASILEIRO DE TERAPIA OCUPACIONAL, 9., 2005, Recife. Anais... Recife: Associação dos Terapeutas Ocupacionais de Pernambuco, 2005. CD-ROM.

SAMPAIO, R. C; GODINHO, K. A Terapia Ocupacional constrói sua prática dentro dos NASFs de Feira de Santana. In: CONGRESSO BRASILEIRO DE TERAPIA OCUPACIONAL, 11., 2009, Fortaleza. Anais... Fortaleza: Associação dos Terapeutas Ocupacionais do Ceará, 2009. CD-ROM.
SILVA, J. A. O Agente Comunitário de Saúde do Projeto QUALIS: agente institucional ou agente de comunidade? 2001. $231 \mathrm{f}$. Tese (Doutorado em Administração Hospitalar)-Faculdade de Saúde Pública da Universidade de São Paulo, São Paulo, 2001.

SOARES, L. B. T.; OLIVEIRA, M. S. O desafio de ser preceptora/facilitadora de terapia ocupacional em aprendizagem significativa: A experiência de formação na residência multiprofissional em saúde da família e comunidade pela UFSCar. In: CONGRESSO BRASILEIRO DE TERAPIA OCUPACIONAL, 10., 2007, Goiânia. Anais... Goiânia: Associação dos Terapeutas Ocupacionais de Goiás, 2007. CD-ROM.

SOUZA, C. C. B. X.; ROCHA, E. F. Portas abertas ou portas fechadas? Acesso à reabilitação nas unidades básicas saúde da região sudeste do município de São Paulo - período de 2000 a 2006. Revista Terapia Ocupacional da Universidade de São Paulo, São Paulo, v. 21, n. 3, p. 230-239, 2010.

SOUZA, C. C. B. X.; ROCHA, E. F. Pensando a terapia ocupacional nos NASFs: a questáo do apoio matricial. In: CONGRESSO BRASILEIRO DE TERAPIA OCUPACIONAL, 11., 2009, Fortaleza. Anais... Fortaleza: Associação dos Terapeutas Ocupacionais do Ceará, 2009. CD-ROM.

\section{Contribuição dos Autores}

Bianca G. Carrasco-Bassi foi responsável pelo levantamento dos trabalhos nos congressos brasileiros de Terapia Ocupacional, autora da dissertação cujas reflexôes integram o presente artigo e das análises e elaboração do texto. Ana Paula S. Malfitano foi orientadora de mestrado da primeira autora e orientadora de iniciação científica da segunda autora, sendo responsável pelas análises, elaboração e redação do texto. Pamela C. Bianchi foi responsável pelo levantamento dos artigos nos periódicos analisados. Todas as autoras participaram da redação e aprovação final do texto.

\section{Notas}

${ }^{1}$ Dados componentes de estudo de mestrado da primeira autora, sob orientação da segunda autora, desenvolvido no Programa de Pós-Graduação em Terapia Ocupacional da Universidade Federal de São Carlos (UFSCar).

${ }^{2}$ A Política Nacional de Atenção Básica (BRASIL, 2006), atualizada através da Portaria n. 2.488, de 21 de outubro de 2011, considera os termos Atenção Básica e Atenção Primária à Saúde termos equivalentes. Nesse artigo, também utilizaremos as duas denominações, embora privilegiemos o termo Atenção Básica em Saúde. Em nossa concepção, ABS aproxima-se mais das açôes básicas de saúde, entendidas aqui como as açóes de saúde relacionadas a partir das demandas de uma determinada população, de acordo com suas características socioculturais e econômicas (MALFITANO; LOPES, 2003).

${ }^{3}$ O Programa de Saúde da Família no município de São Paulo, que teve início em 1996, recebeu a denominação Projeto QUALIS - Qualidade Integral em Saúde - e, embora calcado no modelo dos PSF, apresentava particularidades, por referência à proposta, tanto em relaçáo à forma de gestấo, operacionalizada através de parcerias do estado com organizaçóes sociais, quanto à organização das atividades assistenciais inseridas como nova realidade numa metrópole como São Paulo (SILVA, 2001). 